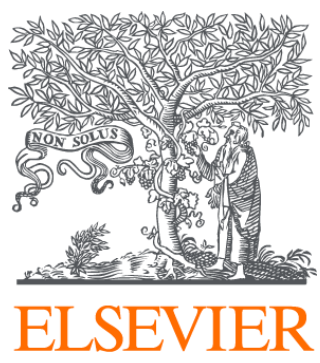

Since January 2020 Elsevier has created a COVID-19 resource centre with free information in English and Mandarin on the novel coronavirus COVID-

19. The COVID-19 resource centre is hosted on Elsevier Connect, the company's public news and information website.

Elsevier hereby grants permission to make all its COVID-19-related research that is available on the COVID-19 resource centre - including this research content - immediately available in PubMed Central and other publicly funded repositories, such as the WHO COVID database with rights for unrestricted research re-use and analyses in any form or by any means with acknowledgement of the original source. These permissions are granted for free by Elsevier for as long as the COVID-19 resource centre remains active. 


\title{
17
}

\section{Beta adrenergic antagonists and antianginal drugs}

\author{
Lindsey M. Stever, PharmD ${ }^{a}, *$, Christopher J. Demitraszek, PharmD, MSCR ${ }^{a}$, \\ Sheena A. Murray, PharmD ${ }^{b}$, and Asima N. Ali, PharmD, BCACP ${ }^{c}$ \\ ${ }^{a}$ Vidant Medical Center, Greenville, NC, United States \\ ${ }^{\mathrm{b}}$ Wilson Medical Center, A Duke LifePoint Hospital, Wilson, NC, United States \\ 'University of Illinois at Chicago College of Pharmacy, Chicago, IL, United States \\ *Corresponding author: lindsey.stever@vidanthealth.com
}

\section{DRUG-DRUG INTERACTIONS}

Patients with mental health disorders are at an increased risk of cardiovascular disease compared to the general population. Therefore, patients may receive medications to treat psychiatric and cardiovascular conditions concomitantly, increasing the risk of drug interactions. To assess the occurrence of adverse drug interactions between antipsychotics and either beta blockers or calcium channel blockers, medication orders from both inpatient and outpatient settings were analyzed by a pharmacotherapy consultant group in Poland. Clinically relevant identified drug interactions are listed in Table 1. Most drug interactions occurred with beta blockers $(n=13)$. The authors note that none of the elucidated drug interactions involving beta blockers have been previously described. The most common adverse effect reported among both beta blockers and calcium channel blockers was cardiac arrythmias. All drug interactions were classified as either probable or certain for causing the noted clinical outcome. Patients analyzed had an average age of $63.13(\mathrm{SD}=7.07)$ and were prescribed an average of six total medications. The authors note that cardiovascular medications and antipsychotics are frequently concomitantly prescribed, therefore clinicians should be mindful of the risk of drug interactions and resultant side effects. Several of the noted drug interactions have unclear mechanisms, therefore further study is needed to add to the body of knowledge regarding the concomitant use of cardiac medications and antipsychotics (Siwek et al., 2020) [c].

\section{BETA-ADRENORECEPTOR ANTAGONISTS [SEDA-38, 173; SEDA-40, 243; SEDA-41, 197; SEDA-42, 195]}

\section{Respiratory}

The Global Initiative for Asthma (GINA) guidelines acknowledge that beta-blockers can cause bronchospasm, however specific recommendations regarding patients with asthma in addition to a chronic condition warranting beta blocker therapy are absent beyond closely monitoring patients. A network meta-analysis was conducted to elucidate the risk of experiencing an asthma attack precipitated by beta blocker therapy. The primary outcome was the incidence of an asthma attack in patients with or without a past medical history of asthma and either receiving a beta blocker or placebo. A total of 24 articles comprising 1301 adult patients were included in the analysis. Patients had an average age of 54.5 (range $22.0-77.3$ ) years and $22.6 \%$ of patients were female. Investigated beta blockers included oral propranolol, oral pindolol, oral atenolol, oral acebutolol, oral sotalol, oral metoprolol, oral practolol, oral oxprenolol, oral timolol, oral nadolol, infusion of sotalol, oral labetalol, oral bisoprolol, oral carvedilol, oral celiprolol, infusion of esmolol, infusion of propranolol, infusion of tolamolol, oral carteolol, infusion of propranolol and labetalol, infusion of practolol, oral celiprolol and propranolol, and oral bevantolol. The authors determined that oral timolol $(\mathrm{RR}=3.35,95 \%$ CI 1.04-10.85) and infused propranolol $(R R=10.19$, 95\% CI 1.29-80.41) were associated with a higher risk 
TABLE 1 Clinically relevant pharmacologic interactions between antipsychotics and/or beta blockers identified by University Center for Clinical Drug Adverse Effects Monitoring and Study at the Clinic of Pharmacology of the Jagiellonian University Medical College.

\begin{tabular}{|c|c|c|c|c|c|}
\hline $\begin{array}{l}\text { Clinical result of drug } \\
\text { interaction }\end{array}$ & $\begin{array}{l}\text { Cardiac } \\
\text { medication }\end{array}$ & Antipsychotic & $\mathbf{n}$ & Proposed mechanism of interaction & $\begin{array}{l}\text { Fatalities } \\
\text { noted (\#) }\end{array}$ \\
\hline \multicolumn{6}{|l|}{ Beta Blockers } \\
\hline Afib & Atenolol & Risperidone & 1 & & 0 \\
\hline Bradycardia & Metoprolol & Perphenazine & 1 & & 0 \\
\hline Ventricular arrhythmia & Metoprolol & Sertindole & 1 & Inhibition of sertindole metabolism via CYP2D6 & 0 \\
\hline Seizure & Metoprolol & Aripiprazole & 1 & Inhibition of aripiprazole metabolism via CYP2D6 & 0 \\
\hline Increased salivation & Metoprolol & Aripiprazole & 2 & Inhibition of aripiprazole metabolism via CYP2D6 & 0 \\
\hline $\begin{array}{l}\text { Myoclonus, urine } \\
\text { retention }\end{array}$ & Metoprolol & Clozapine & 1 & Inhibition of clozapine metabolism via CYP2D6 & 0 \\
\hline Bradycardia & Metoprolol & Perphenazine & 1 & & 0 \\
\hline Syncope & Metoprolol & Chlorprotixene & 1 & & 0 \\
\hline Hypotonia & Nebivolol & Chlorprotixene & 1 & & 0 \\
\hline Ventricular arrhythmia & Sotalol & Ziprasidone & 3 & & 1 \\
\hline \multicolumn{6}{|l|}{ Calcium Channel Blockers } \\
\hline Hypotonia & Amlodipine & Haloperidol & 1 & Inhibition of amlodipine metabolism via CYP3A4 & 0 \\
\hline Ventricular arrhythmia & Diltiazem & Sertindole & 1 & Inhibition of sertindole metabolism via CYP3A4 & 0 \\
\hline Blurred vision, syncope & Diltiazem & Quetiapine & 1 & Inhibition of quetiapine metabolism via CYP3A4 & 0 \\
\hline Hypotonia & Lercanidipine & Haloperidol & 1 & Inhibition of lercanidipine metabolism via CYP3A4 & 0 \\
\hline Bradycardia & Verapamil & Risperidone & 1 & & 0 \\
\hline Restless leg syndrome & Verapamil & Quetiapine & 1 & Inhibition of quetiapine metabolism via CYP3A4 & 0 \\
\hline
\end{tabular}

of bronchospasm in the overall study population compared to placebo. Other agents studied, namely oral celiprolol ( $\mathrm{RR}=0.39,95 \%$ CI 0.044 .11$)$, co-administered oral celiprolol and propranolol ( $R R=0.46,95 \%$ CI $0.02-11.65)$, oral bisoprolol ( $\mathrm{RR}=0.46,95 \% \mathrm{CI} 0.02-11.65)$, oral atenolol $(\mathrm{RR}=0.51,95 \% \mathrm{CI} 0.20-1.28)$, intravenous practolol $(\mathrm{RR}=$ $0.80,95 \%$ CI $0.03-25.14$ ), and intravenous of sotalol $(\mathrm{RR}=0.91,95 \% \mathrm{CI} 0.08-10.65)$ were associated with a lower incidence of asthma attack in the overall patient population compared to placebo. Investigators additionally analyzed risk of asthma attack in patients with a previously diagnosed history of asthma. In this patient population, oral timolol and intravenous propranolol still conferred an increased risk of asthma attack compared to placebo. However, increased incidence of asthma attack in this population was also seen with oral labetalol $(R R=6.60,95 \%$ CI 1.01-43.29), oral oxprenolol $(R R=$ $5.15,95 \%$ CI $1.81-14.69)$, oral propranolol $(R R=3.35$, $95 \%$ CI 1.20-9.38), and oral metoprolol $(R R=3.03,95 \%$ CI 1.09-8.41). The authors conclude that oral timolol and intravenous propranolol increase the risk of bronchospasm in the general patient population and use of these agents should be contraindicated in patients with a history of asthma. This study also provides guidance for the use of beta blocker therapy in patients with a condition warranting therapy and concomitant asthma. Namely, oral formulations of celiprolol, bisoprolol, and atenolol, should be preferentially selected for ambulatory patients (Huang et al., 2021) [M].

\section{ATENOLOL}

\section{Fetotoxicity}

A network meta-analysis was conducted to determine the safety and efficacy of antihypertensive medications in pregnant women with pre-established hypertension (HTN). The primary outcomes of the study were incidence of preeclampsia and small for gestational age (SGA). A total of 22 studies were incorporated in the analysis, which included 4464 women and evaluated treatment with atenolol, amlodipine, pindolol, nifedipine, methyldopa, labetalol, ketanserin, and furosemide. The median age of included patients was 31.3 years, and the baseline systolic blood pressure was reported as $140 \mathrm{mmHg}$ (IQR 136-143). In part, the authors 
determined that the risk of SGA was highest with atenolol $(\mathrm{OR}=26.00,95 \% \mathrm{CI} 2.61-259.29)$ compared to placebo and was assigned a probability score of 0.98 . Additionally, the risk of preterm birth was determined to be increased with atenolol use $(\mathrm{OR}=3.67,95 \% \mathrm{CI}$ 1.29-10.38). Similar risk of preterm birth was observed with labetalol (OR $=10.48,95 \%$ CI 2.71-40.56) and methyldopa $(\mathrm{OR}=6.14,95 \%$ CI 1.72-21.97). The authors concluded that atenolol use was associated with increased risk of SGA and increased rates of cesarean delivery. Future comparative randomized controlled trials should be undertaken to provide clearer guidance for practitioners regarding the safest and most efficacious antihypertensive for use in pregnancy, however, until more data is attained, providers may consider avoiding atenolol in pregnant women with pre-established HTN (Bellos et al., 2020) [M].

\section{BISOPROLOL}

\section{Drug-drug interaction}

While newer oral anticoagulants for nonvalvular atrial fibrillation (NVAF) have fewer cytochrome P450 interactions as compared to warfarin, there are concerns related to drug-drug interactions in relation to P-glycoprotein (PGP) transporters. A pilot study was performed to assess the impact of bisoprolol, a drug with known PGP inhibitor properties commonly used for NVAF, on the plasma levels of dabigatran. Patients in the study were taking dabigatran, with or without concomitant bisoprolol, for at least 2 months prior to plasma levels being obtained. In group 1 ( $n=18$, with bisoprolol) the mean age was $72.39 \pm 8.72$ years, $67 \%$ of patients were women, and the mean $\mathrm{CHA}_{2} \mathrm{DS}_{2} \mathrm{VASc}$ was $3.22 \pm 1.06$. Ten $(56 \%)$ of these patients were taking dabigatran doses of $110 \mathrm{mg}$ twice daily with the remainder taking dabigatran $150 \mathrm{mg}$ twice daily. The group $2(n=11$, without bisoprolol) mean age was $68.64 \pm 10.96,36 \%$ were female, with a mean $\mathrm{CHA}_{2} \mathrm{DS}_{2} \mathrm{VASc}$ of $2.73 \pm 1.66$. Six $(55 \%)$ patients were taking dabigatran $110 \mathrm{mg}$ twice daily and the remainder were on dabigatran $150 \mathrm{mg}$ twice daily. Baseline dabigatran plasma levels and plasma levels at $2 \mathrm{~h}$ following drug administration were found to be significantly higher in patients on concomitant bisoprolol (group 1) as compared to those not on bisoprolol (baseline: $161.35 \pm 95.81$ vs $87.94 \pm 51.77 \mathrm{ng} / \mathrm{mL}$; after $2 \mathrm{~h}$ : $236.87 \pm 119.69$ vs $151.00 \pm 68.88 \mathrm{ng} / \mathrm{mL})$. The authors concluded that an interaction between bisoprolol and dabigatran exists, and while there are no associations made between the increase in plasma drug concentrations and outcomes, this study supports further research to better define the impact these interactions have on clinical outcomes (Nehaj et al., 2020) [c].

\section{CARTEOLOL}

\section{Drug-drug interaction}

An 84-year-old female presented to a hospital with new-onset shortness of breath and chest discomfort. Her home medications and medical history included ophthalmic carteolol and travoprost for glaucoma, azilsartan and doxazosin for CKD, and verapamil (which was recently added) for paroxysmal atrial fibrillation (Afib). At the time of admission, the patient was bradycardic with a heart rate of $29 \mathrm{bpm}$ and profoundly hypotensive with only a palpable radial artery pulse. Laboratory data included elevated serum potassium, liver function tests (LFTs) and lactate levels. An initial 12-lead ECG revealed a heart rate of $24 \mathrm{bpm}$, narrow QRS and type 1 second degree AV block. The carteolol eye drops, along with azilsartan and verapamil, were stopped on arrival and the patient required transvenous pacing until the next day, at which point her bradycardia and AV block resolved. An ophthalmologist was consulted during admission and switched the patient to a non-beta blocker eye drop (dorzolamide hydrochloride). The patient was subsequently discharged home on hospital day 20. The authors conclude that while ophthalmic carteolol is considered to have a lower risk of bradycardia due to having intrinsic sympathomimetic activity, the risk thereof is still present and can increase in the setting of drug-drug interactions with CYP2D6. Close monitoring for bradycardia should be considered for patients at risk for this drug-drug interaction (Arai et al., 2021) [A].

\section{CARVEDILOL}

\section{Endocrine}

A 48-year-old woman with severe HTN and longstanding epigastric pain was found to have a large heterogeneous mass $(9.9 \times 12.7 \times 14.7 \mathrm{~cm})$ in the right upper abdomen and was referred to urology for suspected renal tumor. On a later hospitalization she had a blood pressure of $160 / 90 \mathrm{mmHg}$, heart rate of $120 \mathrm{bpm}$ and was given $50 \mathrm{mg}$ of atenolol. Two hours later her respiratory status deteriorated. Her blood pressure was recorded as $220 / 120 \mathrm{mmHg}$ with a heart rate of $150 \mathrm{bpm}$. She required mechanical ventilatory support with aggressive blood pressure and volume status management due to new cardiomyopathy. Based on the patient's clinical course following the administration of atenolol and large right upper quadrant mass, it was suspected that the patient was experiencing pheochromocytoma crisis and started on prazosin. Biochemical markers of pheochromocytoma later supported this diagnosis and her prazosin was converted to doxazosin. While her blood pressure was 
maintained on doxazosin $8 \mathrm{mg} /$ day, she continued to have a heart rate of $100 \mathrm{bpm}$. Carvedilol was added and gradually up titrated to $25 \mathrm{mg}$ / day to achieve a heart rate of 60-70 beats / $\mathrm{min}$ without reemergence of pheochromocytoma crisis. The authors highlight the importance of having adequate alpha blockade established prior to the addition of beta blockers in patients with who are suspected to have pheochromocytoma. It is also important to note that even beta blockers with dual alpha and beta blocking activity (labetalol and carvedilol) should not be used as the sole drug in blood pressure control as the risk of potentiating hypertensive crisis is high due to the lower alpha blocking abilities of these drugs (Wannachalee \& Chunharojrith, 2020) [A].

\section{Immunologic}

A 74-year-old woman was diagnosed with idiopathic left ventricular failure and started on several medications, including carvedilol. Approximately 1 month later the patient presented with progressive nausea and vomiting, right upper quadrant abdominal pain, and diarrhea. White blood cell count and eosinophils were elevated on admission $(16.7 \times 10(9) / \mathrm{L}$ and $1.5 \times 10(9) / \mathrm{L}$, respectively). The patient developed an acute kidney injury and LFT derangement on day 4 (alkaline phosphatase [ALP] 394U/L, alanine transaminase [ALT] $114 \mathrm{U} / \mathrm{L}$, aspartate transaminase [AST] $79 \mathrm{U} / \mathrm{L}$, and gammaglutamyl transferase [GGT] 145U/L). A peak eosinophil count of $15.8 \times 10(9) / L$ developed on day 5 . The differential for elevated eosinophil count included parasitic infections, vasculitis, and myocarditis; all of which were ruled out. The patient's symptoms improved following cessation of carvedilol and her lab abnormalities normalized 2 months following discharge. Carvedilol was attributed as the probable cause of drug reaction with eosinophils and systemic symptoms (DRESS) given the timing of drug initiation and improvement on discontinuation. The authors state that this was the first published case of DRESS associated with carvedilol and caution clinicians to look beyond the common causes of DRESS when faced with similar scenarios (Jones \& Isenmen, 2020) [A].

\section{ESMOLOL}

\section{Cardiovascular}

Dobutamine stress echocardiography (DSE) has been utilized to identify and prognosticate long-term outcomes related to coronary artery disease (CAD). Inducing cardiovascular stress via pharmacologic methods has generally been done safely although few serious complications have been reported in the literature such as supraventricular or ventricular arrhythmias and myocardial infarction. A case of ST-elevation myocardial infarction (STEMI) during a DSE has been reported following the administration of esmolol. A 53-year-old female developed left-sided chest pain that radiated to the left arm concerning for acute coronary syndrome. The patient did not have a prior history of CAD and it was deemed appropriate to perform a DSE to evaluate for inducible ischemia and presence of CAD. Intravenous dobutamine was titrated up to $50 \mu \mathrm{g} / \mathrm{kg} / \mathrm{min}$ and intravenous atropine $(0.75 \mathrm{mg})$ was given per institutional protocol. The DSE was stopped due to significant tachycardia with QRS widening and intravenous esmolol $15 \mathrm{mg}$ was administered. The patient then began to develop new substernal chest pain with a troponinemia and was treated with sublingual nitroglycerin, aspirin, and ticagrelor. A left heart catheterization did not reveal any evidence of CAD while an ECG demonstrated ST-segment elevations that gradually improved. Takotsubo cardiomyopathy and spontaneous coronary artery dissection were eventually ruled out and the cause of ST elevations was attributed to coronary artery vasospasm. The authors postulated that while the beta- 2 activity of dobutamine was counteracted by esmolol, the weak alpha-1 vasoconstriction-related properties of dobutamine was unopposed and contributed to significant coronary vasospasm. While more studies are needed to confirm the potential for co-administered dobutamine and esmolol to potentiate coronary vasospasm, providers should be aware of this potential risk to streamline differential diagnoses in patients who experience STEMI immediately post DSE (Manasrah et al., 2020) [A].

\section{LABETALOL}

\section{Liver}

A 30-year-old pregnant female (G2P1 at 27 weeks gestation) with a history of HTN and myomectomy was admitted with lower quadrant pain. Aside from an elevated blood pressure of $159 / 92 \mathrm{mmHg}$ and elevated ALT of $206 \mathrm{U} / \mathrm{L}$ and AST of $524 \mathrm{U} / \mathrm{L}$, her exam was benign. The initial differential included severe preeclampsia, hemolysis, elevated liver enzymes, and low platelets (HELLP) syndrome, and acute fatty liver of pregnancy which were ruled out. Oral labetalol was switched to oral nifedipine on hospital day 4 and her liver enzymes ultimately peaked on hospital day 8 to AST of 360U/L and ALT of 1099 U/L. A liver core biopsy reported a normal liver architecture devoid of any derangement that would support the patient's presentation. The patient was discharged on hospital day 12 on oral nifedipine with slowly improving liver enzymes. Nine weeks later at the time of delivery, her liver 
enzymes were nearly within normal limits. Labetalol was ultimately identified as the offending agent due to lack of other supporting evidence for other diagnoses and since labetalol has been shown in other patient populations to cause hepatic injury. The authors conclude that clinicians should be aware of labetalol's adverse effect profile which includes drug-induced liver injury and to not confuse drug-induced liver injury with other conditions which may lead to iatrogenic preterm delivery (Whelan et al., 2020) [A].

\section{TIMOLOL}

\section{Cardiovascular}

Four cases of symptomatic bradycardia with ophthalmic timolol solution use requiring hospitalization are described. The first patient, an 85-year-old male with multiple chronic conditions on amlodipine and tamsulosin, presented to an emergency room (ER) with weakness one day after starting ophthalmic timolol. Electrocardiogram (EKG) showed sinus bradycardia and first-degree heart block. Upon timolol discontinuation, 24-h telemetry showed improvement to normal sinus rhythm. Similarly, a 75-year-old female presented with weakness. Her past medical history was significant for HTN, hyperlipidemia (HLD), dementia, and glaucoma. The patient was noted to be using timolol (duration of treatment was not reported) and concomitantly taking lisinopril. Upon initial presentation, her BP was $59 / 33 \mathrm{mmHg}$ and $\mathrm{HR}$ was 61 BPM. The patient's lisinopril was discontinued and she was discharged to home. After 4 days, the patient presented again with weakness, hypotension (BP of $95 / 66 \mathrm{mmHg}$ ) and a HR of $60 \mathrm{BPM}$. Further questioning revealed onset of symptoms after timolol use. Timolol was discontinued and the patient denied further symptoms of hypotension or bradycardia at a 5-day follow up. A third case describes an 88-year-old female who presented with lightheadedness, palpitations, and near syncope. She was reported to use ophthalmic timolol however her concomitant medications were not reported. The patient was found to be bradycardic with a HR of 47 BPM. After timolol was held, the patient's HR increased, and she had no recurrence of symptoms. The final patient described is a 69-year-old female with onset of dizziness and an episode of syncope 4 days prior to hospitalization. BP on arrival as documented as 146/73 mmHg with a HR of 40-45 BPM. A thorough cardiac and neurological workup was unremarkable. Meclizine was prescribed to alleviate the patient's dizziness, however, her HR remained low. Her timolol eye drops were held during hospitalization and discontinued at discharge. At first outpatient follow-up, the patient reported resolution of dizzy spells. The authors conclude that the elderly population is more susceptible to bradycardia as a result of ophthalmic timolol use for multiple reasons, including pre-existing cardiovascular disease, prolonged pharmacokinetics, and increased potency, emphasizing that the bioavailability of timolol ophthalmic solution is almost equivocal to that of IV timolol. The authors also suggest the use of ophthalmic prostaglandin analogs rather than non-selective beta-blockers (such as timolol) for the treatment of primary open angle glaucoma, especially in the elderly population (Abbas et al., 2020) [A].

\section{NEBIVOLOL}

\section{Sexual function}

Previous evidence indicates that beta blocker monotherapy is less efficacious for blood pressure reduction in Black patients compared to Caucasians. A pilot study evaluated the utility of nebivolol monotherapy in SubSaharan African patients for reducing blood pressure. A total of 140 patients were included in the study, 60 patients were male. The authors note reported side effects were as expected for beta-blockers, including dizziness and headaches. However, male patients also reported episodes of erectile dysfunction $(n=2,3.3 \%$ of male patients). The reported incidence of erectile dysfunction (ED) in this study exceeds the previously reported incidence of $<1 \%$ per nebivolol prescribing information. Follow-up research should be done to determine if beta blocker induced ED is more prevalent than in previous research, or if the patient population studied (Sub-Saharan Africans) are at an increased risk of this side effect (Ojji et al., 2020) [C].

\section{NADOLOL}

\section{Death}

A case study reports the death of an infant after use of oral nadolol for infantile hemangioma (IH). The patient was a 17-week-old healthy, Caucasian female being treated for an $\mathrm{IH}$ on her face. Initial dosing was started at $0.25 \mathrm{mg} / \mathrm{kg}$ BID and titrated up every 4 days for 2 weeks, following currently accepted dosing for this indication. Another dose increase occurred 3 weeks later. Soon after, the patient began having less desire to feed and had not stooled for approximately 10 days. The infant expired 12 days after the last nadolol dose increase. Autopsy could not determine a cause of death. The cardiac blood nadolol concentration was found to be $0.94 \mathrm{mg} / \mathrm{L}$. Of note, serum concentration of nadolol in adult patients taking therapeutic doses was previously determined to 
be $0.077 \pm 0.007 \mathrm{mg} / \mathrm{L}$, however toxic concentrations have not previously been established. The authors recommend propranolol be used as first line therapy for $\mathrm{IH}$, and to have stricter monitoring parameters on behavioral changes in infants receiving nadolol. Stool changes are recommended as a paramount monitoring parameter due to $70 \%$ nadolol elimination unchanged in the gastrointestinal tract (McGillis et al., 2020) [A].

\section{METOPROLOL}

\section{Psychological}

A case details an 84 YOM with multiple medical problems most significant for cardiovascular disease, Afib, aortic valve replacement, and mild dementia; his medication list included metoprolol and amlodipine however concomitant medications were not reported. The patient presented to an acute care facility with a 4-week history of increased confusion, nightmares, fatigue, sleep disturbance, lightheadedness, and gait problems. Six weeks prior he required an ER visit for fatigue and lightheadedness and his amlodipine dose was decreased from $10 \mathrm{mg}$ daily to $5 \mathrm{mg}$ daily due to suspected orthostatic hypotension. The patient reported only mild improvement of fatigue and lightheadedness while his other symptoms continued to worsen. Upon investigation, patient stated the symptoms correlated with metoprolol administration. The metoprolol dose was decreased, and the patient experienced symptom improvement. Symptoms completely resolved after metoprolol was tapered off. Clinicians should be aware that metoprolol and other beta blockers must be used cautiously in patients with pre-existing cognitive dysfunction. If a beta blocker is used in this patient population, the least lipophilic option, such as atenolol, should be considered to reduce the amount of drug that crosses the blood-brain barrier, thus lessening potential psychological adverse effects (Shah et al., 2020) [A].

\section{Electrolyte balance}

A 59-year-old female presented to the ER with chest pain. Her past medical history was significant for type 2 diabetes (T2DM), HTN, and CAD. The patient had been taking metoprolol tartrate $12.5 \mathrm{mg}$ twice daily prior to admission, other medications were not reported. Her primary diagnosis was unstable angina and she was incidentally found to have a serum potassium of $6.7 \mathrm{mEq} / \mathrm{L}$. Treatment for hyperkalemia occurred over multiple days and included calcium gluconate injection, salbutamol nebulization, oral calcium polystyrene sulfonate, insulin, and furosemide injection. Over the course of her hospitalization, her metoprolol dose was increased to $25 \mathrm{mg}$ BID. Despite treatment, after the metoprolol dose was increased, the patient's serum potassium increased to $7.0 \mathrm{mEq} / \mathrm{L}$ on hospital day 2. On day 3, the metoprolol was stopped. By day 5, the potassium decreased to $5.3 \mathrm{mEq} / \mathrm{L}$ and the patient was discharged. This case emphasizes that patients taking metoprolol should undergo routine monitoring for hyperkalemia, especially those with increased potential for electrolyte derangements such as patients with chronic kidney disease and diabetes (Rawal et al., 2020) [A].

\section{Pregnancy}

A retrospective cohort study evaluated 300 pregnant women with chronic HTN living in Iran. Included patients received either methyldopa $250 \mathrm{mg}$ three times a day, amlodipine $5 \mathrm{mg}$ per day or metoprolol $50 \mathrm{mg}$ per day. The mean age of women included was $32.7 \pm 4.78$ years, $31.8 \pm 4.56$ years, and $32.6 \pm 4.69$ years in the metoprolol, methyldopa, and amlodipine groups, respectively. The study outcomes were placental abruption, preeclampsia, parturition eclampsia, perinatal mortality, low birth weight, intrauterine growth restriction (IUGR), and preterm labor. Metoprolol had a higher incidence of low birth weight (15 of 24 cases, $P=0.007$ ), IUGR (10 of 16 cases, $P=0.0032$ ), and preterm labor (22 of 39 cases, $P=0.001)$ compared to the other treatments studied. Side effects were noted for amlodipine (edema, $25 \%$ ) and methyldopa (bronchospasm-induced dyspnea, $10 \%)$. The authors concluded that metoprolol use leads to higher prevalence of low birth weight, IUGR, and preterm labor and amlodipine was associated with fewer adverse pregnancy outcomes. It should be noted that blood pressure control was not evaluated in this study which could have significantly impacted pregnancy outcomes. Additionally, this study's results are hypothesis generating given the small sample size and homogenous patient population, and more studies are needed to confirm the safety of amlodipine during pregnancy (Ghafarzadeh et al., 2020) [C].

\section{Drug-drug interactions}

A single-center, open-label, fixed-sequence, two-treatment crossover study aimed to evaluate, in part, the drugdrug interaction between eliglustat and metoprolol. Metoprolol was chosen as it is a known CYP2D6 substrate. Eliglustat is extensively metabolized by CYP2D6 and previous research suggests it is a moderate CYP2D6 inhibitor. On day 1, metoprolol was administered alone on an empty stomach. Metoprolol and eliglustat twice daily were separately administered on days 3-6. On day 7, metoprolol was co-administered with eliglustat. Treatment day 8 resembled treatment days 3-6. Obtained plasma levels of metoprolol on day-7 compared to day-1 revealed a 1.5-fold higher mean $C_{\max }$ as well as 
a twofold higher area under the curve (AUC), respectively. Eliglustat concentrations on day 7 were at or close to accepted steady state concentrations, confirming in vitro studies showing eliglustat to be a moderate CYP2D6 inhibitor. Implications for tolerability and potential side effects were varied. Five of the 14 subjects receiving metoprolol with eliglustat reported a total of nine mild side effects throughout the different treatment phases. Two subjects reported side effects after metoprolol alone. One subject reported a side effect after only receiving eliglustat. Three subjects reported five adverse effects after receiving the combination of medications. The only side effect reported more than once was headache. The authors gather that with the lack of severe adverse effects, and moderate increase in AUC, doses of CYP2D6 substrate medications used with eliglustat can be decreased but specific dosing recommendations cannot be made with the current data. The authors specify that narrow therapeutic index drugs, tricyclic antidepressants, and phenothiazines should be closely monitored when initiating eliglustat therapy (Thibault et al., 2020) [c].

\section{SOTALOL}

\section{Cardiovascular}

A multicenter analysis evaluated data from five hospitals over a 4-year period regarding sotalol initiation. The aim of the study was to compare different dosing strategies with the goal of creating an outpatient sotalol initiation protocol that would minimize the risk of torsade de pointes ( $\mathrm{tDp})$, a well-known potential adverse effect of sotalol. The authors determined patients who did not require a dosage adjustment during sotalol initiation were more likely to be successfully discharged on sotalol (OR: 6.6, 95\% CI: 1.3-32.7, $P=0.02$ ). Patient characteristics that were associated with not requiring a sotalol dose adjustment included the presence of a pacemaker and concurrent $\beta$-blocker use (OR 0.17 and 0.63 , respectively). While these patient characteristics were associated with successful sotalol initiation, more studies should be done to help devise a specific plan for outpatient sotalol initiation with the goal of reducing the incidence of $\mathrm{tDp}$ (Biswas et al., 2020) [c].

CALCIUM CHANNEL BLOCKERS [SEDA38, 175; SEDA-39, 246; SEDA-40, 243; SEDA41, 199; SEDA-42, 199]

\section{Drug-drug interactions}

Patients with Afib are often co-prescribed anticoagulants and rate control agents. A retrospective, comparative effectiveness cohort study analyzed healthcare claims across the United States to compare potential drug-drug interactions (as evidenced by incidence of overall bleeding) between direct oral anticoagulants (DOACs) and verapamil or diltiazem using active comparators. Patients without documented renal impairment and receiving a DOAC for Afib were included. Rates of bleeds among patients concomitantly receiving diltiazem or verapamil $(n=1793)$ were compared to that of patients receiving concomitant metoprolol $(n=3224)$; rates of bleeds of 1764 patients receiving diltiazem or verapamil (adjusted to capture first rate control agent used) were compared to that of patients receiving amlodipine $(n=3105)$. Patients receiving diltiazem or verapamil in combination with apixaban or rivaroxaban experienced bleeds at a similar rate as patients in comparator groups. However, patients receiving dabigatran and either diltiazem or verapamil were more likely to experience a bleed compared to patients receiving amlodipine (HR 1.52; 95\% CI 1.05-2.20) and patients receiving metoprolol (HR 1.43; $95 \%$ CI 1.02-2.00). The authors conclude that concomitant use of verapamil or diltiazem and dabigatran increases rate of bleeding compared to concomitant use of dabigatran and either metoprolol and amlodipine in patients with unimpaired renal function and Afib. While it is known that P-gp inhibitors, such as diltiazem and verapamil, increase serum concentration of dabigatran, current prescribing information in the United States recommends dose adjustment of dabigatran for this interaction in renally impaired patients only. This study emphasizes the need to monitor patients receiving concomitant dabigatran and diltiazem or verapamil more closely regardless of renal function and to consider alternative DOAC therapy, if possible, to mitigate bleeding risk considering this drug interaction (Pham et al., 2020) [MC].

\section{Mouth and teeth}

While calcium channel blocker-mediated drug induced gingival overgrowth (DIGO) may prompt clinicians to modify antihypertensive therapy to prevent recurrence, the comparative incidence of DIGO among different calcium channel blockers (CCB), as well as other antihypertensives, is not fully known. A cross sectional study evaluating rates and severity of DIGO seen in ambulatory, hypertensive adults receiving either amlodipine, lercanidipine, benidipine, or an ACE inhibitor or ARB, was undertaken at a single center in Turkey. Patients could not have concomitant diabetes, periodontal disease or autoimmune disorders and must have been non-smokers to participate. The average age of the 131 total patients included in the study was 53.7 years, and $52 \%$ of participants were female. DIGO was observed in $19.6 \%$ of patients receiving any $C C B, 12.5 \%$ of patients receiving an ARB and $7.5 \%$ of patients receiving an ACE; however, these rates were not found to be statistically different 
$(P=0.241)$. Of the patients treated with CCB therapy, DIGO was noted in $31.8 \%$ of patients treated with amlodipine, $13.3 \%$ of patients treated with lercanidipine and $7.1 \%$ of patients treated with benidipine. No statistical difference in rates of DIGO among the studied CCB was noted $(P=0.456)$. Amlodipine dose was found to be positively correlated with incidence of DIGO $(r=0.419, P=0.007)$ and women treated with amlodipine were more likely to experience DIGO compared to men (13 vs 9 patients, respectively; $P<0.001$ ). Duration of therapy, patient age, and oral hygiene were not noted to affect DIGO rates. Of the patients who experienced DIGO, no difference was found in the severity of this adverse effect. The authors concluded that patients are at equal likelihood of experiencing DIGO while taking either a CCB, ACE, or ARB. It should be noted that external validity of these results is severely limited by small sample size and homogenous patient population. Based off the results of this study, if DIGO is a concern when treating ambulatory hypertensive adults, an alternative antihypertensive to amlodipine in female patients or patients requiring higher doses is preferred (Ustaoğlu et al., 2020) [C].

\section{AMLODIPINE}

\section{Mouth and teeth}

John and colleagues describe a case of a 19 YOM patient with HTN and stage 5 chronic kidney disease of unknown etiology who presented with painless swelling of his gums that began and gradually progressed 3 months after initiating amlodipine $10 \mathrm{mg}$ daily. Laboratory analyses were not consistent with blood dyscrasias; therefore, the authors preliminarily diagnosed the patient with DIGO and discontinued amlodipine antihypertensive therapy in favor of atenolol and prazosin. At the patient's 6 month follow up, his gingival overgrowth (GO) had completely resolved. The authors deferred re-challenging the patient with amlodipine to definitively diagnose DIGO, however, per a WHO-UMC causality assessment, amlodipine was determined to be the "probable/likely" cause of the patient's symptoms. The authors conclude that amlodipine can cause GO that is potentially reversable if therapy is discontinued. It should be noted that a similar case describes GO severe enough to cause dental malposition requiring multiple extractions (Quenel et al., 2020) [A]. Similarly, Quach and colleagues describe a case of amlodipine-induced GO that was not apparent until the patient received dental implants. The patient described is a 72-year-old Caucasian female with a past medical history significant for squamous cell carcinoma in the floor of the mouth and mandible (resulting in mandibulectomy and requiring mandibular dental implants), as well as HTN. The patient was taking amlodipine; however, duration of therapy and concomitant medications were not discussed. After receiving dental implants, the patient presented with GO surrounding them. The GO was excised and biopsied to exclude recurrence of the patient's malignancy. Four months following excision, the patient experienced a recurrence of GO surrounding her implant. The authors determined that the patient's GO was induced by amlodipine and this therapy was discontinued. The patient's overgrowth was excised, and she remained free of recurrence. The authors hypothesized that the patient's implant precipitated oral biofilm formation which triggered GO in addition to the patient's amlodipine. They emphasize meticulous oral hygiene as well as mechanical removal of biofilms to prevent GO in patients with dental implants. Both cases highlight the importance of obtaining a thorough medication history to determine potential drug-induced causes of new patient findings ( John et al., 2020 [A]; Quach \& Ray-Chaudhuri, 2020 [A]).

While GO is a known potential side effect of calcium channel blocker therapy, the mechanism of DIGO is not completely understood. A single-center study aimed to determine if local concentrations of transforming growth factor-b1 (TGF- $\beta 1$ ), platelet-derived growth factor-BB (PDGF-BB), and basic fibroblast growth factor (bFGF) in gingival crevicular fluid (GCF) differed between patients experiencing amlodipine-induced GO, in the same patients at sites without clinical GO, and in healthy controls. Three adult female and two adult male patients with moderate to severe amlodipine-induced GO (mean age 53.4 years) as well as three adult female and two adult male healthy patients (mean age 53.0 years) were enrolled. Samples of GCF were taken in 56 sites with clinical $\mathrm{GO}(\mathrm{GO}+)$, in 38 sites from patients with GO but in areas with healthy gingiva $(\mathrm{GO}-)$, and from 45 sites of the healthy patients (control). The authors determined that the concentration of TGF- $\beta 1$ in GO+ sites $(9.50 \pm 7.3 \mathrm{ng} / \mathrm{mL})$ was not statistically different than in GO - sites $(2.07 \pm 0.50 \mathrm{ng} / \mathrm{mL}, P=0.067)$ but was significantly higher compared to control samples $(2.74 \pm 1.01 \mathrm{ng} / \mathrm{mL}, P=0.005)$. Concentrations of PDGF$\mathrm{BB}$ did not differ between the three groups. The authors note that bFGF was not detected in enough samples to perform statistical analysis. Given the difference in TGF- $\beta 1$ concentrations, the authors propose amlodipine use may increase expression of TGF- $\beta 1$, which in turn promotes gingival extracellular matrix deposition and matrix synthesis. This study is hindered by a small sample size and additional investigation is needed to confirm results. However, case and control patients were similar in age and sex which possibly allowed patients to be otherwise more clinically similar (Kose et al., 2020) [c].

A similar study sought to determine if the presence of IL17-A in the tissues of patients could distinguish 
amlodipine-induced GO from inflammatory gingival enlargement and normal, healthy gums. Non-smoking individuals between the ages of 20 and 60 years were enrolled and included patients with GO and receiving amlodipine therapy $(n=9)$, patients with chronic inflammatory gingival enlargement $(n=11)$, and patients with clinically healthy gingiva as a control group $(n=9)$. It should be noted that the mean age of patients in the amlodipine group was significantly higher than the other groups studied (57.7 years vs 26.18 and 26.55 years, respectively, $P=0.0000003$ ). Blood and gingival tissue samples were collected from all participants and assessed for IL17-A expression. The investigators determined that IL17-A expression was significantly higher in the gingival tissue of the amlodipine treated patients than in the patients with chronic gingival inflammation (81.9 vs 66.08; $P<0.05)$ and the control group patients $(81.9 \mathrm{vs}$ 66.08; $P<0.05)$. No difference was found in the IL17-A expression in the serum samples from all three groups. The authors hypothesize that amlodipine may contribute to IL17-A synthesis, leading to gingival fibrosis that is independent of inflammation, however, they note that more studies are needed to further elucidate the mechanism by which amlodipine causes GO. It should be noted that in this study, amlodipine treated patients were significantly older than patients included in the other study groups which may indicate a higher burden of other chronic diseases, a potential confounder (Sume et al., 2020) [c].

\section{Fluid balance}

Peripheral edema is a common side effect of amlodipine therapy; however, it is unclear if there is a genetic predisposition to this deleterious effect. A study enrolling 240 Han Chinese patients from two outpatient sites sought to test if any variants of CYP3A5 were associated with amlodipine-induced peripheral edema. The included patients all received amlodipine for at least four weeks prior to enrollment. Patients experiencing peripheral edema with WHO-UMC causality ratings of certain, probable, or possible for amlodipine as the cause $(n=64)$ were categorized as cases and the remaining patients were considered controls. Cases were more likely to be female $(P=0.00048)$ and patients who self-reported drinking alcohol were more likely to be controls $(P=0.034)$. No other differences in baseline characteristics between cases and controls were noted. The authors found three alleles that appeared protective against amlodipine induced peripheral edema and were more common in the control group: rs15524 $\mathrm{G}$ (vs $\mathrm{A}, \mathrm{OR}=0.53, P=0.011$ ), rs $4646453 \mathrm{~A}$ (vs $\mathrm{C}, \mathrm{OR}=0.54, P=0.019$ ) and $\mathrm{rs} 776746$ $\mathrm{T}$ (vs $\mathrm{C}, \mathrm{OR}$ 0.58. $P=0.03$ ). The associated $\mathrm{A}, \mathrm{C}$, and $\mathrm{C}$ alleles were more common in the case group. The TT + CT genotype of rs776746 was also demonstrated to be protective from amlodipine-induced peripheral edema after controlling for patient gender and alcohol consumption status (TT $+\mathrm{CT}$ vs $\mathrm{CC}$ : $\mathrm{OR}=0.57$ adjusted $P=0.044$ ). The authors determined that the risk of amlodipineinduced peripheral edema may be associated with CYP3A5 genetic polymorphisms in the Han Chinese population. While this study has limited external validity due to homogenous patient population and small sample size, the data contributes to the growing knowledge regarding the utility of individualized medicine (Liang et al., 2021) [c].

\section{Liver}

A 30 YOM with a past medical history significant for HTN and a kidney transplant from a living donor five years prior presented to an outpatient clinic with abdominal distension, pain, and weight gain. The patient's medication regimen included tacrolimus, mycophenolic acid, prednisolone, acetylsalicylic acid, carvedilol, clopidogrel, and amlodipine. Duration of amlodipine use was not reported however the dose was reported as $10 \mathrm{mg}$ daily. Abdominal ultrasound was notable for free fluid in the abdomen but was otherwise normal. The ascitic fluid was evaluated and determined to have total protein of $0.8 \mathrm{~g} / \mathrm{L}$, albumin $0.7 \mathrm{~g} / \mathrm{L}$ and low density lipoprotein (LDL) 26U/L. The patient's corresponding serum albumin was $4.4 \mathrm{~g} / \mathrm{dL}$, total protein $6.9 \mathrm{~g} / \mathrm{dL}$, urea $46 \mathrm{mg} /$ $\mathrm{dL}$ and creatinine $2.63 \mathrm{mg} / \mathrm{dL}$. LFTs were within normal ranges; INR was 0.96. All other imaging studies were unremarkable, and the patient's ascites was determined to have an etiology other than liver dysfunction. Calcium channel blocker mediated arterial dilation was suspected and amlodipine was discontinued. Within one week, a reduction in abdominal fluid was noted and the patient's ascites completely resolved after two months. The authors conclude that amlodipine caused this patient's ascites; Naranjo scale was not reported. Additional studies are needed to elucidate the exact mechanism by which amlodipine can cause ascites, as well as any association with duration of therapy. Clinicians should be aware of this rare side effect when evaluating new ascites in a patient with otherwise unremarkable liver workup and taking amlodipine (Arasan et al., 2020) [A].

A 47 YOM with a past medical history significant for untreated HTN presented to an acute care facility with hemorrhagic stroke. Upon presentation, the patient's LFTs, electrolytes, and coagulation profile were reported as normal. After initial management, the patient was started on amlodipine $5 \mathrm{mg}$ daily on hospital day four. His concomitant medications on hospital day six included enalapril, subcutaneous enoxaparin, and vitamin D2 orally weekly. On hospital day seven, routine laboratory analysis revealed ALT of $449 \mathrm{U} / \mathrm{L}$, AST $271 \mathrm{U} / \mathrm{L}$, total bilirubin $15 \mu \mathrm{mol} / \mathrm{L}$ and INR 1.2. Viral hepatitis 
serologies were negative. Drug induced liver injury (DILI) was suspected, and amlodipine was discontinued. The patient's LFTs improved after five days off amlodipine and returned to baseline after four weeks off amlodipine. The probability the patient experienced DILI was rated as "probably" per the Council for International Organization of Medical Sciences and the Roussel Uclaf Causality Assessment Method. The authors concluded that amlodipine was the most likely cause of the patient's DILI as his transaminases increased after commencement of amlodipine therapy and improved after amlodipine was discontinued. They do note that as amlodipine is not considered a hepatotoxic medication and amlodipine induced DILI is very rarely reported, additional studies are needed to help quantify the rate of DILI secondary to amlodipine use (Varghese et al., 2020) [A].

\section{Musculoskeletal}

The 2017 ACC/AHA hypertension guidelines recommend caution when prescribing thiazide diuretics to treat HTN in patients with a history of gout (Whelton et al., 2018) [S]. However, guidance on antihypertensive selection to mitigate gout risk is not provided. A secondary analysis of the ALLHAT trial (ALLHAT Collaborative Research Group, 2002) [C] was conducted to determine the effect of treatment with amlodipine, lisinopril, or chlorthalidone on Medicare gout claims among participants aged 65 years or older living in the United States. The analysis included 23964 patients who were 69.8 years of age on average at baseline and followed for an average of 4.9 years; $45 \%$ of the participants were women and $31 \%$ self-identified as non-Hispanic Black. The authors state baseline characteristics were similar among all three treatment arms. A total of 597 individual patients were linked to a gout claim after enrollment in ALLHAT. The 5-year incidence of gout was determined to be 1.59 per 100 person years for patients treated with amlodipine, 2.47 per 100 person years for chlorthalidone, and 1.93 per 100 person years for lisinopril. Amlodipine significantly reduced the risk of gout compared to both chlorthalidone (HR 0.63; 95\% CI: 0.51-0.78) and lisinopril (HR 0.74; 95\% CI: 0.58-0.94). Results were similar when stratified for age, sex, race, baseline atenolol use, and history of T2DM, CKD, CVD, and self-reported estrogen use. The authors concluded that antihypertensive treatment with amlodipine was associated with a lower gout risk than treatment with lisinopril or chlorthalidone. Although this analysis included a large, diverse patient population, the results do require additional study to identify patients that most benefit from amlodipine therapy, as the participant's baseline gout history, concomitant use of uric acid-lowering therapy, and alcohol consumption were not assessed, nor were uric acid levels obtained during treatment (Juraschek et al., 2020) [R].

\section{Drug overdose}

The Swedish Poison Center evaluated all amlodipine overdose consult requests received from hospitals in a two-year time frame to determine the incidence of noncardiogenic pulmonary edema (NCPE) in severe amlodipine poisoning. This prospective observational study enrolled a total of 19 patients who deliberately ingested amlodipine, required treatment with vasopressors or inotropes, and had a blood amlodipine concentration of at least $0.25 \mu \mathrm{g} / \mathrm{mL}$ at presentation (median concentration was $0.47 \mu \mathrm{g} / \mathrm{mL}$ ). Included patients had a median age of 60 years and $47 \%$ were female. The presence of NCPE was determined by the need for positive pressure ventilation that was not better explained by another cause in a patient with a left ventricular ejection fraction of at least $50 \%$. Patients who expired $(n=4)$ or required VA-ECMO $(n=1)$ were not considered to meet criteria for NCPE. Of the nine patients $(47 \%)$ who met criteria for NCPE, four required intubation. All nine patients were treated with high dose insulin and made full recoveries. The authors concluded that NCPE is common in amlodipine poisoning, occurring in approximately half of the reported cases. Additionally, the authors comment that high dose insulin infusions may increase the risk of NCPE by contributing to volume overload and by increasing cardiac output. Therefore, the authors recommend positive pressure ventilation, concentrated dextrose infusions (D50W) to prevent hypoglycemia, and diuretics, as needed, to supplement high dose insulin in patients with amlodipine overdose; they advise against increasing the insulin infusion dose, as well as the administration of albumin which may exacerbate volume overload. Given the small cohort of this study, further evaluation of NCPE in amlodipine poisoning is likely needed to determine optimal therapy (Lindeman et al., 2020) [c].

\section{Drug-drug interactions}

Prescribing information for amlodipine, a CYP3A4 substrate, states that amlodipine pharmacokinetics are not significantly altered by the representative moderate CYP3A4 inhibitor erythromycin. Hibino and colleagues describe a case of a patient concomitantly receiving aprepitant, a CYP3A4 inhibitor, and amlodipine and subsequently developing a second-degree atrioventricular block. The described patient was a 73-year-old male being treated with cisplatin, gemcitabine, and an antiepidermal growth factor receptor monoclonal antibody for lung cancer with supportive aprepitant to prevent nausea and vomiting. The patient's other pertinent past medical history included HTN and first-degree atrioventricular block. Five days after concomitantly taking amlodipine and aprepitant, the patient experienced a Mobitz type I block and amlodipine was discontinued. 
After $24 \mathrm{~h}$, the patient's EKG returned to baseline. The probability of amlodipine causing the new second degree heart block was rated as "probable" by the Naranjo scale as well as the Drug Interaction Probability Scale. The authors conclude that the CYP3A4 interaction between amlodipine and aprepitant likely contributed to the patient's new heart block. This case highlights the importance of diligent patient profile review for all drug interactions, as the interaction between aprepitant and amlodipine is considered "moderate" and would unlikely prompt preemptive therapy modification to avoid the interaction (Hibino et al., 2021) [A].

\section{Special review}

The COVID-19 pandemic, described as the public health crisis of this generation, has prompted evaluation of previously approved therapies on this disease's course, progression, and mortality. Zhang and colleagues evaluated antihypertensive therapies in 96 patients receiving similar concomitant treatment with antiviral therapy, corticosteroids, respiratory support, traditional Chinese medicine, and antibiotics on COVID-19 mortality rates at a single academic medical center in Wuhan, China. Patients were admitted to the hospital for COVID-19 symptoms and were included in the analysis if HTN was the sole comorbidity. Of the included patients, 19 received amlodipine, 14 received nifedipine, 8 received an ACEI or ARB, 10 patients did not receive any antihypertension therapy inpatient and 45 had indeterminate antihypertension therapy. Patients were of similar age (average 66.5 years), gender distribution ( $45 \%$ female), day from symptom onset (day 10 on average), experienced similar COVID-19 symptoms and had similar blood pressure on admission between all 5 groups ( $P>0.05$ for all baseline demographics). The fatality rate of patients who did not receive amlodipine was 19.5\%, compared to $0 \%$ among patients treated with amlodipine $(P=0.037)$. ICU admission and use of invasive ventilation did not differ with antihypertensive treatment. The authors hypothesize that regulation of intracellular calcium by calcium channel blockers inhibits viral mechanisms of inflammation, such as viral replication, mitochondrial dysfunction, and cell death. The authors concluded that amlodipine therapy is associated with decreased COVID-19 mortality among patients with concomitant HTN. It should be noted that this study did not evaluate dose or duration of amlodipine therapy, and external validity is limited by small sample size and homogenous patient ethnicity (Zhang et al., 2020) [c].

Solaimanzadeh similarly conducted a retrospective review of 65 patients admitted to a community hospital in Brooklyn, NY who tested positive for SARS-CoV-2 and were aged 65 years or older. Patients were stratified as either receiving amlodipine or nifedipine during their hospitalization $(n=24)$ or not receiving calcium channel blocker therapy $(n=41)$. Patients were enrolled if they had concomitant conditions other than HTN, such as diabetes or asthma, however baseline demographics did not differ between the two patient groups. Use of antibiotics, anticoagulants, steroids, and hydroxychloroquine therapy also did not differ between the two groups. Use of antiviral therapy was not assessed. The fatality rate of patients who received calcium channel blocker therapy was 50\%, compared to $85.4 \%$ among patients who did not receive calcium channel blocker therapy $(P=0.0036)$. Patients who received calcium channel blocker therapy were less likely to receive mechanical ventilation $(4.2 \%$ vs $39.0 \% ; P=0.0026)$. Outcomes of patients treated with amlodipine and nifedipine were not distinguished, and total duration of therapy could not be assessed. The author concluded that nifedipine or amlodipine therapy is associated with improved mortality in elderly patients hospitalized with COVID-19. Like the Zhang study, significant limitations to external validity exist with small sample size and single enrollment center. Additionally, the use of hydroxychloroquine, now disfavored as a treatment for COVID-19, and lack of background antiviral therapy are potential confounders (Solaimanzadeh, 2020) [c].

While further, rigorous study is needed to confirm the effect of calcium channel blocker therapy on COVID-19 mortality, given the current data and in the absence of contraindications or prevailing comorbidities, clinicians could consider amlodipine as antihypertensive therapy for patients hospitalized with COVID-19 to possibly reduce COVID-19 mortality.

\section{BENIDIPINE}

\section{Liver}

A 69-year-old male patient presented to an acute care facility with asymptomatic acute hepatitis and was diagnosed with IgG4-related autoimmune hepatitis (AIH) possibly secondary to benidipine use. The patient had a past medical history significant for HTN, T2DM, prior alcohol abuse, and hyperuricemia; he had begun treatment with benidipine 2 months prior to developing hepatitis. His concomitant medications included sitagliptin, febuxostat, and insulin glargine. Prior to initiating benidipine, the patient's LFTs were within normal limits. On admission, the patient's total bilirubin was $2.7 \mathrm{mg} / \mathrm{dL}$; his AST, ALT and ALP were all greater than five times the upper limit of normal. The patient's viral hepatitis serology was negative. Notably, his IgG was $3158 \mathrm{mg}$ / $\mathrm{dL}$ and $\mathrm{IgG} 4$ was $703 \mathrm{mg} / \mathrm{dL}$. All other laboratory data and imaging studies were unremarkable. Pathology findings were consistent with chronic active hepatitis due to AIH. The patient scored 14 on the 1999 revised original AIH scoring system and 8 on the simplified criteria for the diagnosis of AID, respectively corresponding to a "probable" and "definite" diagnosis of AIH. Lymphocytic infiltration with an IgG4/IgG-positive cell ratio of $60 \%$ was seen on immunohistochemistry, consistent with 
IgG4-related AIH. Benidipine therapy was withdrawn, however after 2 weeks, the patient's liver function tests did not improve, and the patient was started on prednisolone $40 \mathrm{mg}$ orally daily and tapered over five months. Remission was rapidly achieved after starting prednisolone and maintained on ursodiol $600 \mathrm{mg}$ daily once prednisolone taper was completed. Naranjo scale for benidipine as the precipitating agent for the patient's hepatitis was not reported. The authors conclude that druginduced IgG4-related $\mathrm{AIH}$ is rare and difficult to definitively diagnose. More data is needed to correlate cases of IgG4-related AIH to diagnostic criteria and to elucidate the most effective treatment regimens for patients experiencing this adverse reaction (Kawabata et al., 2020) [A].

\section{DILTIAZEM}

\section{Cardiovascular}

Topically applied vasodilators are used during coronary artery bypass grafting (CABG) to prevent graft spasm. There is a paucity of data comparing rates of vascular lesions and atherosclerosis due to topical vasodilator selection. A prospective, randomized, case control study was conducted in a single center in Brazil to compare the rates of endothelial apoptosis seen in left internal thoracic artery (LITA) grafts as a surrogate marker for the risk of vascular lesions after exposure to topical papaverine, diltiazem and nitroprusside. Patients undergoing elective CABG $(n=10)$ were enrolled; seven patients were male, and the median age was 65.5 years. LITA tissue samples were obtained from each patient and divided into four segments. A sample from each patient was exposed to a control solution $(0.9 \% \mathrm{NaCl})$, diltiazem, nitroprusside, and papaverine. Apoptosis was determined using the terminal deoxynucleotidyl transferasemediated dUTP-biotin nick end labeling (TUNEL) technique. Apoptotic cell count was determined by taking the average count from 50 randomly selected areas. Tissue exposed to the control solution had the lowest average number of apoptotic cells ( $4.25 \pm 1.4$ cells), followed by nitroprusside (9.48 \pm 2.09 cells), and papaverine $(10.75 \pm 2.37$ cells). Tissue exposed to diltiazem had the most apoptotic cells (13.3 \pm 2.8 cells). Rates of apoptosis was significantly different between all four groups $(P=0.0001)$. The authors determined that all vasodilators studied caused apoptosis, with diltiazem having the most pronounced effect. They conclude that the evidence they obtained should be weighed when determining risks and benefits of topical vasodilator selection. It should be noted that this study's small sample size, short term results, and lack of long-term clinical correlation limit external validity. Additional studies are necessary to definitively link apoptosis rates with clinical outcomes, in addition to determining the most clinically favorable topical vasodilator for the prevention of graft spasm during CABG (Unal et al., 2020) [E].

\section{Ear, nose, throat}

An 85-year-old woman developed an acute inflammatory mass after incompletely swallowing an extendedrelease (ER) diltiazem tablet. The patient's notable past medical history included Afib, heart failure with reduced ejection fraction, chronic obstructive pulmonary disease, and tobacco abuse. The patient presented to the ER approximately $18 \mathrm{~h}$ after incompletely swallowing a diltiazem ER tablet. The dose of the tablet was not reported. Physical exam revealed mild stridor, neck swelling, and tenderness to palpation. The patient was achieving $100 \%$ oxygen saturation on room air. Computerized tomography of the neck was read as "heterogenous mass-like peripherally enhancing lesion within the right pre-epiglottic fat". The radiologist determined the mass to be approximately $18 \mathrm{~mm}$ long and, given the patient's history of tobacco abuse, suspected malignancy. The patient was treated with IV dexamethasone (dose not reported) and discharged with otolaryngologist follow up, a course of amoxicillin-clavulanic acid, and pain control with liquid hydrocodone. The patient reported complete resolution of her symptoms after two days which was confirmed by video laryngoscopy. The authors determined that the patient's localized inflammation was caused by retaining an ER diltiazem tablet in the esophagus. As this patient's inflammation was selflimiting and did not require airway protection, the authors conclude that close observation may be sufficient for patients who present with a similar clinical scenario. Providers should be mindful of this rare drug-induced cause of airway inflammation as it may not require intensive interventions, highlighting the necessity of obtaining a thorough medication history upon patient presentation (Manning \& Roth, 2020) [A].

\section{Skin}

Diltiazem use can rarely cause photo-distributed hyperpigmentation. A case describes a 71-year-old woman with Fitzpatrick type V skin who presented to a dermatology clinic with dark brown, velvety-textured, symmetric patches distributed across her face. Notably, the patient had been prescribed diltiazem $100 \mathrm{mg}$ daily six months prior to presenting. Her past medical history was significant for HTN, HLD, T2DM, and seasonal allergies. Her concomitant medications included losartan, 
hydrochlorothiazide, spironolactone, furosemide, pravastatin, aspirin, metformin, pioglitazone, and fluticasone (doses not reported). A biopsy obtained from one of the hyperpigmented areas was read as vacuolar interface change with pigment incontinence. The patient was diagnosed with diltiazem-associated hyperpigmentation. A Naranjo scale to determine likelihood of diltiazem as the cause of the patient's findings was not reported. Diltiazem was discontinued and the patient was treated with a 3-month course of tacrolimus $0.1 \%$ ointment and hydroquinone $4 \%$ cream. The patient's hyperpigmented areas were noted to be significantly improved at her 8 -month follow up. The authors highlight the importance of early recognition of drug-induced dermatological adverse effects to avoid patient distress (Siegel \& Ko, 2020) [A].

\section{FELODIPINE}

\section{Gastrointestinal}

One hallmark histopathological finding in patients with severe celiac disease $(\mathrm{CD})$ is villous atrophy of the duodenal mucosa. As CYP3A4 is in duodenal villous epithelial and hepatic parenchymal cells, Chretien and colleagues sought to determine if severity of $C D$ affects systemic concentration of drugs that undergo substantial first pass metabolism via CYP3A4. A total of 115 patients (47 with CD and 68 healthy patients) were enrolled from a single site in Ontario to participate in the open-label, single-dose, pharmacokinetic study. Included patients ranged in age from 18 to 83 years old and 64 patients were male. Participants in the $\mathrm{CD}$ group were stratified by severity of disease according to the modified MarshOberhuber (M-O) classification. All patients received one felodipine $10 \mathrm{mg}$ ER tablet and underwent serial blood draws. The healthy patients repeated felodipine ingestion in a randomized, crossover design; once taken with water and once taken with grapefruit juice, a CYP3A4 inhibitor. Plasma samples were assessed for felodipine concentrations. Patients with severe $C D$ $\left(\mathrm{M}-\mathrm{O}\right.$ scores $3 \mathrm{~b}$ and $3 \mathrm{c}$ ) had similar felodipine $\mathrm{AUC}_{0-\infty}$ concentrations as healthy patients who ingested felodipine with grapefruit juice $(36.9 \pm 5.6$ vs $36.8 \pm 3.5 \mathrm{ngh} / \mathrm{mL}$, respectively), which significantly differed from patients with $\mathrm{CD}$ but normal duodenal mucosa (M-O score 0, $\left.\mathrm{AUC}_{0-\infty} \quad 20.5 \pm 4.5 \mathrm{ngh} / \mathrm{mL} ; \quad P<0.05\right)$ and healthy patients that ingested felodipine with water $\left(\mathrm{AUC}_{0-\infty}\right.$ $16.3 \pm 1.4 \mathrm{ngh} / \mathrm{mL} ; P$ 0.0001). Clinical implications of differences in felodipine concentrations, such as blood pressure, were not assessed. The authors concluded that patients with severe CD are potentially at risk for either adverse effects or inadequate response to medications metabolized by CYP3A4 and recommended therapeutic alternatives that are not metabolized by CYP3A4 for these patients. It should be noted that further study is needed to elucidate the differences in clinical response to medications metabolized by CYP3A4 in patients with CD (Chretien et al., 2020) [c].

\section{NICARDIPINE}

\section{Urinary tract}

Data from the 1000 patients enrolled in the ATACH 2 trial (Qureshi et al., 2016) [MC] was analyzed to determine the incidence and precipitating factors of acute kidney injury (AKI) in patients with intracerebral hemorrhage. Patients who participated in the ATACH 2 trial had serum creatinine measurements at baseline, 24, 48, and $72 \mathrm{~h}$; AKI was defined by the investigators by the AKIN classification. The area under the curve (AUC) of nicardipine over $24 \mathrm{~h}$ was determined to be positively correlated with incidence of AKI (RR 1.004; $P<0.001$ ) and renal adverse events (RR 1.006; $P<0.001)$. The authors speculate that larger recorded doses of nicardipine may reflect more difficult to treat HTN and therefore may indirectly identify patients more likely to experience poorer clinical outcomes. Alternatively, nicardipine may be directly decreasing renal vascular resistance and decreasing GFR. In the studied patient population, AKI was associated with increased risk of death or disability at 90 days and therefore further studies are warranted. It should be noted that the conclusions of this study are hypothesis generating as they resulted from a post-hoc analysis. The authors did not provide any guidance in ameliorating risk of AKI as a result of nicardipine use (Qureshi et al., 2020) [MC].

\section{NIFEDIPINE}

\section{Cardiovascular}

A retrospective case-control study was conducted to determine if the use of dihydropyridine calcium channel blockers (DHP CCB) confer a risk of out-of-hospital cardiac arrest (OHCA). Two cohorts of adult patients were identified from OHCA registries in the Netherlands and Denmark who were taking either amlodipine or nifedipine for at least 90 days prior to a ventricular tachycardia/ventricular fibrillation (VT/VF) event leading to an OHCA. Doses of the two medications of interest were categorized as "high" if nifedipine total daily dose (TDD) was $60 \mathrm{mg}$ or higher and amlodipine TDD was $10 \mathrm{mg}$ or 
higher; doses below those thresholds were categorized as "low". Each identified case was matched with up to five patients of the same age and sex who experienced an OHCA but were not concomitantly taking a DHP CCB. It should be noted that the ER formulation of nifedipine was most prescribed ( $n=161$ of 167 total patients prescribed nifedipine). Among the Netherlands cohort, patients taking nifedipine were more likely to experience OHCA compared to patients not taking a DHP $\mathrm{CCB}(\mathrm{OR}=1.28$, CI 1.003-1.63); this risk was driven by patients taking high dose nifedipine (high dose $\mathrm{OR}=1.45$, CI 1.02-2.07; low dose $\mathrm{OR}=1.15$, CI 0.84-1.59). Conversely, amlodipine use, regardless of dose, was shown to be protective against OHCA compared to no DHP CCB use (OR =0.63, CI 0.50-0.79). Similar results were seen in the Denmark cohort; while nifedipine use was not associated with an increased risk of OHCA compared to no DHP CCB use, an increased risk was seen in patients taking high dose nifedipine $(\mathrm{OR}=1.96$, CI 1.18-3.25). Amlodipine use conferred a protective effect on OHCA in the Denmark cohort $(\mathrm{OR}=0.89$, CI 0.82-0.97). There was no statistically significant difference in concomitant medication use among DHP CCB users and non-users in both cohorts. The authors concluded that high dose nifedipine was associated with an increased risk of OHCA and suggest caution when titrating nifedipine doses. Additional studies with a larger population of patients taking nifedipine are needed to assess if careful patient selection can mitigate risk of OHCA when prescribing nifedipine. Based on the results of this study, clinicians can consider the use of amlodipine rather than nifedipine to reduce the risk of OHCA in patients requiring a DHP CCB (Eroglu et al., 2020) [MC].

\section{Pregnancy}

Soares and Gilson describe a case of cardiogenic shock in a pregnant 31-year-old woman secondary to nifedipine tocolysis. The G6P2 woman was 18 weeks pregnant and had a past medical history significant for aortic stenosis. The patient received oral nifedipine 20 and $10 \mathrm{mg}$ over $30 \mathrm{~min}$ and subsequently developed hypotension (BP $82 / 37 \mathrm{mmHg}$ ), chest pain, and diffuse ST-segment depressions on EKG. A troponin peak to $4.822 \mathrm{ng} / \mathrm{mL}$ was noted. Following fluid resuscitation and vasopressor support, the patient's blood pressure and EKG returned to baseline, and her chest pain resolved. The outcome of the pregnancy, as well as any concomitant medications were not documented. The authors postulate that nifedipine-induced vasodilation required a compensatory increase in cardiac output, leading to ischemia secondary to increased myocardial oxygen demand in the setting of fixed obstruction caused by the patient's aortic stenosis. They emphasize following up on any physical exam findings that may indicate aortic stenosis and, if present, to use an alternative tocolytic agent to nifedipine if needed, such as indomethacin (Soares \& Gilson, 2020) $[\mathrm{A}]$.

\section{Drug-drug interactions}

Prescribing information for nifedipine, a CYP3A4 substrate, recommends clinically monitoring patients and considering dose reduction when it is co-administered with a CYP3A4 inhibitor, however, practical guidance for clinicians is lacking. Physiologically based pharmacokinetic (PBPK) modelling was employed to elucidate the interaction between three formulations of nifedipine: immediate release (IR), sustained release (SR) and controlled release (CR), and the CYP3A4 inhibitor ritonavir. Nifedipine IR was modelled as $10 \mathrm{mg}$ twice daily, SR as $30 \mathrm{mg}$ twice daily and CR as $60 \mathrm{mg}$ daily. The dose of ritonavir used was $100 \mathrm{mg}$ twice daily. The PBPK model was linked to pharmacodynamic data to determine the change in systolic blood pressure (SBP) expected from changes in nifedipine's pharmacokinetics due to the interaction with ritonavir. Modelling determined the $\mathrm{C}_{\max }$ $(\mathrm{ng} / \mathrm{mL})$ of nifedipine IR to be 91.00 (43.41-162.80). In the presence of ritonavir, the $C_{\max }$ increased to 1684.18 (208.59-4638.07); the $\mathrm{C}_{\max }$ ratio was determined to be 17.92 (3.94-40.75). Interaction with ritonavir was predicted to decrease SBP by $33.2 \mathrm{mmHg}$. The $\mathrm{C}_{\max }(\mathrm{ng} / \mathrm{mL})$ of nifedipine SR was determined to be 103.44 (36.40-210.04) and 4818.92 (462.87-12171.97) alone and when coadministered with ritonavir, respectively. The resultant $\mathrm{C}_{\max }$ ratio was 43.62 (7.68-93.48), and the interaction with ritonavir predicted a decrease in SBP by $23.04 \mathrm{mmHg}$. Similarly, the $C_{\max }(\mathrm{ng} / \mathrm{mL})$ of nifedipine $\mathrm{CR}$ alone and co-administered with ritonavir were 61.27 (20.87-141.77) and 030.89 (237.21-8340.14); this resulted in a $C_{\max }$ ratio of 8.85 (7.40-106.68) and a decrease in SBP by $23.18 \mathrm{mmHg}$. The therapeutic concentration range of nifedipine is reported as $25-100 \mathrm{ng} / \mathrm{mL}$. The authors report modelling reduced doses of nifedipine (such as nifedipine IR $1.25 \mathrm{mg}$ ) did not significantly alter results. When the model accounted for ritonavir discontinuation with continued use of nifedipine, the plasma concentrations of nifedipine returned to baseline within 5-8 days. The authors concluded that coadministration of nifedipine with ritonavir could potentially result in critical hypotension, even at reduced nifedipine doses. They advise against coadministration of nifedipine and ritonavir and to allow a washout period of at least 5 days from ritonavir discontinuation to re-commencement of nifedipine therapy. It should be noted that this study does not consider the effect of CYP3A4 polymorphisms on the reported drug interaction (Niu et al., 2020) [E]. 


\section{INHIBITORS OF FATTY ACID OXIDATION [SEDA-42, 203]}

\section{Perhexiline}

\section{Sensory systems}

A 71-year-old man was incidentally found to have asymptomatic bilateral optic disc swelling on routine optometry exam. Physical exam findings and imaging were otherwise unremarkable. Cerebrospinal fluid exam showed an opening pressure of $24.5 \mathrm{~cm}$ CSF with no other pertinent findings. Inflammatory and infectious workup were negative. The patient otherwise had a past medical history of hypertrophic cardiomyopathy and ischemic heart disease and had been treated with perhexiline maleate $150 \mathrm{mg}$ daily for two years prior to presentation (the patient's other medications were not reported). A diagnosis of perhexiline-induced optic neuropathy was made and perhexiline was discontinued. Optical coherence tomography retinal nerve fiber layer noted subclinical improvement one month after perhexiline discontinuation. The patient's disc swelling completely resolved eight months later. Notably, the patient was later started on amiodarone $200 \mathrm{mg}$ daily, which resulted in recurrence of optic neuropathy. The authors conclude that patients taking perhexiline or amiodarone should have regular optometry follow up to monitor for adverse ocular effects. Should findings become apparent on ocular exam, the authors recommend discontinuing the offending medication, if possible. Additionally, the authors report resolution of symptoms should be apparent six weeks after discontinuing perhexiline and approximately 14 weeks after stopping amiodarone (Tan et al., 2021) [A].

\section{TRIMETAZIDINE}

\section{Neuromuscular function}

A systematic review was conducted to determine if a time relationship exists between trimetazidine (TMZ) therapy and the appearance of TMZ-induced parkinsonian symptoms, as well as the effect of TMZ withdrawal. A total of five European studies enrolling a total of 88 patients with TMZ-induced parkinsonism were included. Patients ages ranged from 64 to 91 years while treatment duration prior to parkinsonian symptom onset ranged from 1 month to 20 years. One study included patients with subclinical parkinsonism prior to TMZ therapy which worsened when therapy was initiated. Included patients were most likely to report symmetrical akinesia, rigidity, and postural instability and gait disorder and less likely to report tremor. Parkinsonism symptoms in TMZ patients were noted to be mild in severity (MDS-UPDRS Part III scores 10.5 \pm 19 ). After withdrawal of TMZ therapy, 49 patients $(55.7 \%)$ had complete resolution of symptoms. All patients achieved symptom improvement with TMZ withdrawal. The authors state increasing age may be a cofactor in developing TMZ-induced parkinsonism, however, it should be noted that TMZ is usually prescribed in patients with CAD and the age of onset of TMZ-induced parkinsonism may also be reflective of patient's concomitant disease. The authors conclude that TMZ induced parkinsonian symptoms are reversable by withdrawing TMZ therapy. They emphasize careful evaluation of patients taking TMZ, especially elderly patients, for signs of drug induced parkinsonism; if clinical findings are consistent with parkinsonism, they note anti-parkinsonian medications do not alleviate symptoms unless patients had Parkinson's disease at baseline and recommend TMZ withdrawal to alleviate symptoms. This review is hindered by including a variety of study types which limited statistical analysis. Therefore, this review should be considered as hypothesis generating and more studies are needed to further elucidate the clinical course of trimetazidine induced parkinsonism (Dy et al., 2020) [M].

To investigate the incidence of TMZ-associated parkinsonism, a retrospective cohort study evaluated 14-years of claims data from South Korea's National Health Insurance Service-National Sample Cohort (NHIS-NSC). A total of 656524 adult patients were included in the analysis: 9712 patients newly prescribed TMZ during the predefined "exposure ascertainment period" and 29116 matched controls. Claims data were then assessed for the following 7 years (follow up period) for ICD-10 codes related to parkinsonism and doses of TMZ. Patients taking TMZ were divided into three groups according to their determined cumulative defined daily dose (cDDD): patients with less than $7 \mathrm{cDDD}$; with 7-30 cDDD and patients with more than $30 \mathrm{cDDD}$. Included patients were 52.22 years of age on average and $65.29 \%$ of patients were female. The authors found the overall incidence of parkinsonism to be 7.37 per 1000 person years. The incidence was found to be significantly higher in patients taking TMZ compared to controls (9.34 vs 6.71 per 1000 person-years, $P<0.0001)$. This correlated to a HR of $1.38(95 \% \mathrm{CI}=1.26-1.15)$ for parkinsonism in patients taking TMZ. Cumulative incidence of parkinsonism increased in patients taking TMZ as the CDDD increased $(P<0.001)$. Male patients were less likely to have a diagnosis of parkinsonism, while patients over the age of 50 years as well as patients concomitantly diagnosed with diabetes, end state renal disease, or stroke were more likely to have a parkinsonism diagnosis during the follow up period. Additionally, patients with claims for other medication associated with Parkinson-like side effects, such as antipsychotics, prokinetics, and calcium channel blockers, during the follow up period were also more 
likely to be diagnosed with parkinsonism, and the effect was shown to be additive. For patients taking TMZ and one concurrent parkinsonism-inducing drug, the incidence rate of parkinsonism was 8.400 (adjusted $\mathrm{HR}=2.30$, 95\% CI 1.80-2.93), while patients taking TMZ and three or more other parkinsonism-inducing drugs, experienced an increased incidence of 21.125 per 1000 person years (adjusted $\mathrm{HR}=4.58,95 \%$ CI 3.16 6.64). The authors concluded that Korean patients taking TMZ are at an increased risk of developing parkinsonism compared to the Korean population overall. This study's large patient population and relatively long follow up period allow for an in-depth analysis; however, the homogenous patient population may limit external validity. Clinicians should be aware of TMZ-induced parkinsonism, especially in older patients taking multiple medications, as these patients are at a higher risk of this side effect (Kim et al., 2020) [MC].

A prospective, longitudinal study in a single center in Hungary aimed to evaluate the effect of TMZ in patients with pre-existing Parkinson's disease (PD). A total of 42 patients (23 males) diagnosed with PD by the UK Brain Bank criteria and taking TMZ were enrolled and underwent neurologic assessments before and 3 months after discontinuing TMZ therapy. Patients were then followed for 12 months for safety. Included patients were, on average, aged 71 years, had been diagnosed with PD 3.3 years prior to inclusion in the study, and had been taking TMZ for $78.5 \pm 24.9$ months (average daily dose $72.1 \pm 9.8 \mathrm{mg}$ ). Compared to baseline assessment, all four parts of the Hungarian validated version of the Movement Disorders Society-sponsored Unified Parkinson's Disease Rating Scale (MDS-UPDRS), a measure of PD disease severity, significantly improved after TMZ discontinuation (Part I: $15.0 \pm 7.1$ vs $10.9 \pm 6.6$, $P<0.001$; part II: $13.1 \pm 8.4$ vs $9.6 \pm 6.9, P<0.001$; part III: $38.2 \pm 14.4$ vs $27.8 \pm 13.5, P<0.001$; and part IV: $3.4 \pm 3.2$ vs $2.2 \pm 2.4, P=0.004$ ). Improvements on all parts of the MDS-UPDRS met minimal clinically important difference (MCID) threshold values. Postural instability and gait difficulty (PIGD) also significantly improved with TMZ discontinuation $(7.5 \pm 5.2$ vs $10.6 \pm 6.2$ at baseline, $P<0.001$ ). Non-Motor Symptoms Scale (NMSS) scores improved from baseline after stopping TMZ for sleep $(14.3 \pm 8.9$ vs $10.1 \pm 7.3, P=0.001)$, gastrointestinal problems ( $5.5 \pm 8.0$ vs $3.1 \pm 5.2, P=0.024)$, and the total NMSS score $(62.8 \pm 49.9$ vs $46.6 \pm 35.3, P=0.004)$. Scores on the Montgomery-Asberg Depression Rating Scale (MADRS) also improved with TMZ discontinuation ( $14.3 \pm 8.8$ vs $11.4 \pm 6.4, P=0.001$ ); this improvement did reach MCID values. Health related quality of life (HRQoL) was assessed with the 39-item PD Questionnaire (PDQ-39). While follow up values for mobility, stigma, social support, and the summary index all statistically improved compared to baseline, the improvement did not reach
MCID thresholds. It should be noted that despite discontinuing the patient's TMZ therapy, no cardiovascular events were reported during the 12-month follow up. The authors conclude TMZ therapy should be avoided in patients with PD. Given that several scales used to measure severity of PD improved beyond MCID thresholds, the authors recommend discontinuing TMZ and continuing anti-Parkinson's therapies in patients with PD and taking TMZ. Given the study's small sample size and homogenous patient population, there is potential for limits to the result's external validity and results should be confirmed using a wider patient population (Pintér et al., 2020) [c].

\section{NITRATES [SEDA-34, 305; SEDA-35, 354; SEDA-38, 176; SEDA-40, 243]}

\section{Isosorbide dinitrate}

\section{Pregnancy}

There is a lack of data evaluating the efficacy and safety of combination isosorbide-oxytocin in the medical removal of the uterus after intrauterine fetal death (IUFD). A prospective, randomized, double blind, controlled trial sought to compare efficacy and safety of cervical isosorbide dinitrate gel-oxytocin to misoprostol geloxytocin in management of IUFD. Pregnant females beyond 20 weeks gestation were enrolled ( $n=60$, average age approximately 27 years) in the study. Baseline measurements were balanced except for birthweight which was higher in the isosorbide dinitrate-oxytocin group (1251.5g vs $717 \mathrm{~g}$, respectively, $P=0.003$ ). Isosorbide dinitrate $(80 \mathrm{mg} / 1.5 \mathrm{~mL}, n=30)$ or misoprostol $(100 \mu \mathrm{g} /$ $1.5 \mathrm{~mL}, n=30$ ) gel solutions were administered for up to 4 doses or when the Bishop score was $\geq 7$ then oxytocin was given intravenously until uterus expulsion. Rate of fetal evacuation was 4.4 times higher in the isosorbide dinitrate-oxytocin group (HR 4.4; 95\% CI 2.1-9, $P<0.001)$. A reduction in systolic (SBP) and diastolic blood pressure (DBP) between isosorbide dinitrate and misoprostol was seen after the second dose, SBP difference: $7.6 \mathrm{mmHg}(95 \% \mathrm{CI} 6.5-8.7, P<0.001)$ and DBP difference: $6.1 \mathrm{mmHg}(95 \%$ CI $4.3-7.9 \mathrm{mmHg}$, $P<0.001)$. An elevation in HR was noted between the two groups after the second dose (HR difference: $9.5 \mathrm{bpm}(95 \%$ CI $6.7-12.2 \mathrm{bpm})$. An elevation of temperature was seen after initial misoprostol dose $\left(0.1-0.6^{\circ} \mathrm{C}\right.$, $P<0.001)$. The misoprostol group experienced more pelvic pain (RR 8.6; 95\% CI 2.3-35.4, $P=0.001$ ). Alternatively, more patients in the isosorbide dinitrateoxytocin group experienced headaches (RR 1.5; 95\% CI, 0.5-4.8, $P=0.7$ ) and while findings were not statistically significant, they may be clinically relevant. No cases of hemorrhage, uterine tachysystole, hypertonicity, or 
coagulopathy were reported. The authors concluded that isosorbide dinitrate-oxytocin was effective and safe when compared to misoprostol-oxytocin. This study provides new information regarding use of combination isosorbide dinitrate-oxytocin for management of IUFD. Side effects described are previously known (e.g. headache, reductions in BP, and increase in pulse) thus adding to the current body of literature. Study limitations include: small sample size, inclusion of pregnancies 20 weeks post gestation only and exclusion of higher risk populations. Therefore, at this time, the authors recommend utilizing proven alternatives (e.g. prostaglandin analogs, mifepristone) for pregnancy loss until more robust, supportive studies become available. Results may help support further research in this potentially ethically challenging field (Arteaga-Troncoso et al., 2019) [c].

\section{Nitroglycerin}

\section{Skin}

A recent systematic review and meta-analysis was conducted to evaluate the efficacy and safety of topical nitroglycerin in preventing mastectomy flap necrosis (flap failure). This review adds to the growing body of evidence supporting a favorable safety profile of topical nitroglycerin. Investigators included three randomized placebo-controlled trials and two retrospective cohorts which included use of nitroglycerin ointment, at doses $4.5 \mathrm{mg}, 15 \mathrm{mg}$, and $45 \mathrm{mg}$, and $50 \mathrm{mg} / 8 \mathrm{~h}$ transdermal patch. Three studies used one time application of nitroglycerin. Meta-analysis results demonstrated lower flap necrosis rates in the nitroglycerin group (OR $0.23 ; 95 \%$ CI $0.10-0.53, P<0.001$ and $\left.I^{2} 73 P=0.005\right)$. Side effects were evaluated in four studies as a secondary objective. Commonly reported side effects included dizziness, hypotension, and headache however no significant difference was found between groups. Applicability of data is limited as only five studies were included, two being retrospective analyses which cannot prove causality. Also, the variety of formulations, doses, and frequency of use obscures application to practice. However, given the positive safety profile the authors recommend that topical nitroglycerin may be considered as a safe option in the prevention of mastectomy flap necrosis, but doses and products should be limited to those studied due to unknown safety profile of other formulations and strengths (Vania et al., 2020) [M].

\section{References}

Abbas, S. A., Hamadani, S. M., et al. (2020). Ophthalmic timolol and hospitalization for symptomatic bradycardia and syncope: A case series. Cureus, 12(3), e7270 [A].

ALLHAT Officers and Coordinators for the ALLHAT Collaborative Research Group. The Antihypertensive and Lipid-Lowering
Treatment to Prevent Heart Attack Trial. (2002). Major outcomes in high-risk hypertensive patients randomized to angiotensinconverting enzyme inhibitor or calcium channel blocker vs diuretic: The Antihypertensive and Lipid-Lowering Treatment to Prevent Heart Attack Trial (Allhat). JAMA, 288(23), 2981-2997 [C].

Arai, R., Fukamachi, D., et al. (2021). Bradycardia shock caused by the combined use of carteolol eye drops and verapamil in an elderly patient with atrial fibrillation and chronic kidney disease. Internal Medicine (Tokyo, Japan), 60(1), 79-83 [A].

Arasan, S. N., Yenigun, E. C., et al. (2020). Amlodipine induced massive ascites, a rare clinical case. Iranian Journal of Kidney Diseases, 14(6), 517-519 [A].

Arteaga-Troncoso, G., Chacon-Calderon, A. E., et al. (2019). A randomized controlled trial comparing isosorbide dinitrateoxytocin versus misoprostol-oxytocin at management of foetal intrauterine death. PLoS ONE, 14(11), e0215718 [c].

Bellos, I., Pergialiotis, V., et al. (2020). Comparative efficacy and safety of oral antihypertensive agents in pregnant women with chronic hypertension: A network metaanalysis. American Journal of Obstetrics and Gynecology, 223(4), 525-537 [M].

Biswas, M., Levy, A., et al. (2020). Multicenter analysis of dosing protocols for sotalol initiation. Journal of Cardiovascular Pharmacology and Therapeutics, 25(3), 212-218 [c].

Chretien, M. L., Bailey, D. G., et al. (2020). Severity of coeliac disease and clinical management study when using a CYP3A4 metabolised medication: A phase I pharmacokinetic study. BMJ Open, 10(3), e034086 [c].

Dy, A. M. B., Limjoco, L. L. G., et al. (2020). Trimetazidine-induced parkinsonism: A systematic review. Frontiers in Neurology, 11, 44 [M].

Eroglu, T. E., Mohr, G. H., et al. (2020). Differential effects on outof-hospital cardiac arrest of dihydropyridines: Real-world data from population-based cohorts across two European countries. European Heart Journal —Cardiovascular Pharmacotherapy, 6(6), 347-355 [MC].

Ghafarzadeh, M., Shakarami, A., et al. (2020). The comparison of side effects of methyldopa, amlodipine, and metoprolol in pregnant women with chronic hypertension. Hypertension in Pregnancy, 39(3), 314-318 [C].

Hibino, H., Makino, Y., et al. (2021). Exacerbation of atrioventricular block associated with concomitant use of amlodipine and aprepitant in a lung cancer patient: A case report. International Journal of Clinical Pharmacology and Therapeutics, 59(04), 328-332. Online ahead of print. [A].

Huang, K.-Y., Tseng, P.-T., et al. (2021). Do beta-adrenergic blocking agents increase asthma exacerbation? A network meta-analysis of randomized controlled trials. Scientific Reports, 11(1), 452 [M].

John, K., Mishra, A. K., et al. (2020). Amlodipine-induced gingival hyperplasia in a young male with stage 5 chronic kidney disease. Case Reports in Nephrology, 2020, 7801546 [A].

Jones, L., \& Isenmen, H. (2020). Not the usual suspects: DRESS secondary to carvedilol. The New Zealand Medical Journal (Online), 133(1523), 104-105 [A].

Juraschek, S. P., Simpson, L. M., et al. (2020). The effects of antihypertensive class on gout in older adults: Secondary analysis of the antihypertensive and lipid-lowering treatment to prevent heart attack trial. Journal of Hypertension, 38(5), 954-960 [R].

Kawabata, H., Kawakatsu, Y., et al. (2020). Igg4-related autoimmune hepatitis with a suspected drug-induced etiology. Internal Medicine (Tokyo, Japan), 59(11), 1401-1405 [A].

Kim, S., Yu, Y. M., et al. (2020). Trimetazidine use and the risk of parkinsonism: A nationwide population-based study. International Journal of Environmental Research and Public Health, 17(19), 7256 [MC].

Kose, K. N., Yilmaz, S., et al. (2020). The gingival crevicular fluid levels of growth factors in patients with amlodipine-induced gingival overgrowth: A pilot study. Nigerian Journal of Clinical Practice, 23(4), 561-567 [c]. 
Liang, H., Zhang, X., et al. (2021). Association of cyp3a5 gene polymorphisms and amlodipine-induced peripheral edema in Chinese han patients with essential hypertension. Pharmacogenomics and Personalized Medicine, 14, 189-197 [c].

Lindeman, E., Ålebring, J., et al. (2020). The unknown known: Noncardiogenic pulmonary edema in amlodipine poisoning, a cohort study. Clinical Toxicology (Philadelphia, Pa.), 58(11), 1042-1049 [c].

Manasrah, N., Naik, R., et al. (2020). Coronary vasospasm after Dobutamine stress echocardiogram triggered by Esmolol. Cureus, 12(8), e10015 [A].

Manning, W., \& Roth, B. S. (2020). Acute airway inflammation caused by incomplete ingestion of extended-release diltiazem: A case report. The Journal of Emergency Medicine, 58(6), e247-e249 [A].

McGillis, E., Baumann, T., et al. (2020). Death associated with nadolol for infantile hemangioma: A case for improving safety. Pediatrics, 145(1), e20191035 [A].

Nehaj, F., Sokol, J., et al. (2020). Effect of Bisoprolol on the level of dabigatran. American Journal of Therapeutics, 27(2), e159-e164 [c].

$\mathrm{Niu}, \mathrm{W}$., Li, S., et al. (2020). Investigating the interaction between nifedipine- and ritonavir-containing antiviral regimens: A physiologically based pharmacokinetic/pharmacodynamic analysis. British Journal of Clinical Pharmacology, 87(7), 2790-2806. Online ahead of print. [E].

Ojji, D., Ale, B. M., et al. (2020). The effect of nebivolol on office blood pressure of blacks residing in sub-saharan Africa(a pilot study). Frontiers in Cardiovascular Medicine, 7, 613917 [C].

Pham, P., Schmidt, S., et al. (2020). Association of oral anticoagulants and verapamil or diltiazem with adverse bleeding events in patients with nonvalvular atrial fibrillation and normal kidney function. JAMA Network Open, 3(4), e203593 [MC].

Pintér, D., Juhász, A., et al. (2020). The impact of trimetazidine on disease severity and quality of life in parkinson's disease. Scientific Reports, 10(1), 10050 [c].

Quach, H., \& Ray-Chaudhuri, A. (2020). Calcium channel blocker induced gingival enlargement following implant placement in a fibula free flap reconstruction of the mandible: A case report. International Journal of Implant Dentistry, 6(1), 47 [A].

Quenel, L., Keribin, P., et al. (2020). Amlodipine-induced gingival enlargement: A case report. Journal of Stomatology, Oral and Maxillofacial Surgery, 121(3), 308-311 [A].

Qureshi, A. I., Huang, W., et al. (2020). Systolic blood pressure reduction and acute kidney injury in intracerebral hemorrhage. Stroke, 51(10), 3030-3038 [MC].

Qureshi, A. I., Palesch, Y. Y., et al. (2016). Intensive blood-pressure lowering in patients with acute cerebral hemorrhage. New England Journal of Medicine, 375(11), 1033-1043 [MC].

Rawal, K. B., Chhetri, D. R., et al. (2020). Metoprolol-induced hyperkalemia-A case report. Indian Journal of Medical Sciences, 1-4 [A].

Shah, R., Babar, A., et al. (2020). Metoprolol-associated central nervous system complications. Cureus, 12(5), e8236 [A].
Siegel, J. D., \& Ko, C. J. (2020). Diltiazem-associated photodistributed hyperpigmentation. The Yale Journal of Biology and Medicine, 93(1), 45-47 [A].

Siwek, M., Woroń, J., et al. (2020). Adverse effects of interactions between antipsychotics and medications used in the treatment of cardiovascular disorders. Pharmacological Reports, 72(2), 350-359 [c].

Soares, C., \& Gilson, M. (2020). Nifedipine-induced shock in pregnancy with aortic stenosis. Annals of Internal Medicine, 172(3), 221 [A].

Solaimanzadeh, I. (2020). Nifedipine and amlodipine are associated with improved mortality and decreased risk for intubation and mechanical ventilation in elderly patients hospitalized for covid-19. Cureus, 12(5), e8069 [c].

Sume, S. S., Berker, E., et al. (2020). Elevated interleukin-17A expression in amlodipine-induced gingival overgrowth. Journal of Periodontal Research, 55(5), 613-621 [c].

Tan, Y., Sia, P., et al. (2021). Optic neuropathy secondary to perhexiline and amiodarone. BMJ Case Reports, 14(1), e237727 [A].

Thibault, N., Ibrahim, J., et al. (2020). Effect of eliglustat on the pharmacokinetics of digoxin, metoprolol, and oral contraceptives and absorption of eliglustat when coadministered with acid-reducing agents. Molecular Genetics and Metabolism, 129(4), 278-285 [c].

Unal, O., Ulukan, M. O., et al. (2020). Comparison of the apoptotic effects of topically applied papaverine, diltiazem, and nitroprusside to internal thoracic artery. Brazilian Journal of Cardiovascular Surgery, 35(5), 626-633 [E].

Ustaoğlu, G., Erdal, E., et al. (2020). Influence of different antihypertensive drugs on gingival overgrowth: A cross-sectional study in a Turkish population. Oral Diseases, 27(5), 1313-1319. Online ahead of print $[C]$.

Vania, R., Pranata, R., et al. (2020). Topical Nitroglycerin is associated with a reduced mastectomy skin flap necrosis-systematic review and meta-analysis. Journal of Plastic, Reconstructive \& Aesthetic Surgery, 73(6), 1050-1059 [M].

Varghese, G., Madi, L., et al. (2020). A possible increase in liver enzymes due to amlodipine: A case report. SAGE Open Medical Case Reports, 8, 2050313X20917822 [A].

Wannachalee, T., \& Chunharojrith, P. (2020). Safety of preoperative carvedilol in a patient with recent atenolol-induced pheochromocytoma crisis and cardiomyopathy: A case report. Annals of Medicine and Surgery (2012), 60, 360-364 [A]

Whelan, A., Izewski, J., et al. (2020). Labetalol-induced hepatotoxicity during pregnancy: A case report. AJP Reports, 10(3), e210-e212 [A].

Whelton, P. K., Carey, R. M., et al. (2018). 2017 acc/aha/aapa/abc/ acpm/ags/apha/ash/aspc/nma/pcna guideline for the prevention, detection, evaluation, and management of high blood pressure in adults: A report of the American college of cardiology/ American heart association task force on clinical practice guidelines. Hypertension (Dallas, Tex.: 1979), 71(6), e13-e115 [S].

Zhang, L.-K., Sun, Y., et al. (2020). Calcium channel blocker amlodipine besylate therapy is associated with reduced case fatality rate of COVID-19 patients with hypertension. Cell Discovery, 6(1), 96 [c]. 